\title{
Nadolol plus isosorbide mononitrate alone or associated with band ligation in the prevention of recurrent bleeding: a multicentre randomised controlled trial
}

\author{
J C García-Pagán, ${ }^{1,2}$ C Villanueva, ${ }^{2,3}$ A Albillos, ${ }^{2,4}$ R Bañares, ${ }^{2,5}$ R Morillas, ${ }^{2,6}$ \\ J G Abraldes, ${ }^{1,2}$ J Bosch, ${ }^{1,2}$ on behalf of the Spanish Variceal Bleeding Study Group
}

See Commentary, p 1046

${ }^{1}$ Hepatic Hemodynamic Laboratory, Liver Unit, Institut de Malalties Digestives i Metaboliques, Hospital Clínic, Institut de Investigacions Biomèdiques August Pi i Sunyer, Barcelona, Spain; ${ }^{2}$ Centro de Investigación Biomédica en Red de Enfermedades Hepáticas y Digestivas (Ciberehd), Barcelona, Madrid, Spain; ${ }^{3}$ Department of Gastroenterology, Hospital Sant Pau, Barcelona, Spain;

${ }^{4}$ Department of

Gastroenterology, Hospital Universitario Ramón y Cajal, University of Alcalá, Madrid, Spain; ${ }^{5}$ Department of Gastroenterology, Hospital General Universitario Gregorio Marañón, Madrid, Spain;

${ }^{6}$ Department of

Gastroenterology, Hospital

Universitari Germans Trias y

Pujol, Badalona, Spain

Correspondence to:

Dr J Bosch, Hepatic

Hemodynamic Laboratory, Liver Unit, Hospital Clinic, C Villaroel 170, Barcelona 08036, Spain: jbosch@clinic.ub.es

Members of the Spanish Variceal Bleeding Study Group are given at the end of the paper.

Revised 15 December 2008 Accepted 20 January 2009

Published Online First

12 February 2009

\section{ABSTRACT}

Background and aims: Previous clinical trials suggest that adding non-selective beta-blockers improves the efficacy of endoscopic band ligation (EBL) in the prevention of recurrent bleeding, but no study has evaluated whether EBL improves the efficacy of betablockers + isosorbide-5-mononitrate. The present study was aimed at evaluating this issue in a multicentre randomised controlled trial (RCT) and to correlate changes in hepatic venous pressure gradient (HVPG) during treatment with clinical outcomes

Methods: 158 patients with cirrhosis, admitted because of variceal bleeding, were randomised to receive nadolol+isosorbide-5-mononitrate alone (Drug: $n=78$ ) or combined with EBL (Drug+EBL; $n=80$ ). HVPG measurements were performed at randomisation and after 4-6 weeks on medical therapy.

Results: Median follow-up was 15 months. One-year probability of recurrent bleeding was similar in both groups (33\% vs $26 \%$ : $p=0.3$ ). There were no significant differences in survival or need of rescue shunts. Overall adverse events or those requiring hospital admission were significantly more frequent in the Drug+EBL group. Recurrent bleeding was significantly more frequent in HVPG non-responders than in responders (HVPG reduction $\geqslant 20 \%$ or $\leqslant 12 \mathrm{~mm} \mathrm{Hg}$ ). Among non-responders recurrent bleeding was similar in patients treated with Drugs or Drugs+EBL.

Conclusions: Adding EBL to pharmacological treatment did not reduce recurrent bleeding, the need for rescue therapy, or mortality, and was associated with more adverse events. Furthermore, associating EBL to drug therapy did not reduce the high rebleeding risk of HVPG non-responders.

ISRCTN26221020

Patients surviving a first episode of variceal bleeding have a risk of over $60 \%$ of experiencing recurrent haemorrhage within 2 years from the index episode. ${ }^{1}$ As a consequence, all patients surviving a variceal bleed must receive active treatments to prevent rebleeding. ${ }^{1}$ A meta-analysis of the four reported studies comparing nonselective beta-blockers (BB) plus isosorbide-5mononitrate (IsMn) versus endoscopic variceal band ligation $(E B L)^{2-5}$ showed no significant differences between these treatments in both preventing rebleeding and in mortality. Based on this evidence, the 2005 Baveno IV consensus conference recommended either pharmacological therapy with a $\mathrm{BB} \pm \mathrm{IsMn}$ or $\mathrm{EBL}$ as first-line treatments to prevent rebleeding in patients with cirrhosis. ${ }^{6}$ On the other hand, two randomised trials have shown that continued nadolol administration improved the efficacy of EBL in preventing rebleeding, ${ }^{78}$ but did not decrease mortality. Based in these two studies, recent guidelines have recommended $\mathrm{BB}$ plus EBL as the best option for the secondary prevention of variceal haemorrhage. ${ }^{910}$ However, these trials were underpowered and one study was prematurely stopped after an interim analysis despite the absence of a predetermined stopping rule. ${ }^{8}$ In addition, preliminary data from a third study failed to confirm the benefit of combining EBL + drugs, ${ }^{11}$ and so far no study has evaluated whether the addition of EBL improves the efficacy of BB+IsMn.

A reduction in the hepatic venous pressure gradient (HVPG) of at least $20 \%$ of baseline values or to values of $12 \mathrm{~mm} \mathrm{Hg}$ or below is associated with a very low risk of rebleeding during followup. ${ }^{12-14}$ In patients achieving these targets (around $40 \%$ of those receiving $\mathrm{BB}+\mathrm{IsMn}^{2}{ }^{15-17}$ ), adding $\mathrm{EBL}$ is unlikely to enhance the beneficial effect of $\mathrm{BB}$, but certainly will increase the rate of adverse events, so the overall benefit remains conjectural.

Therefore, the present study was aimed at investigating, in a multicentre randomised controlled trial (RCT), whether the association of EBL to pharmacological therapy with nadolol+IsMn is more effective than nadolol+IsMn alone in preventing recurrent upper gastrointestinal bleeding in patients with cirrhosis surviving an episode of variceal bleeding and to establish the correlation between changes in HVPG caused by therapy and the clinical outcome of patients receiving or not EBL on top of drug therapy.

\section{METHODS}

\section{Selection of patients}

This prospective RCT was conducted in six hospitals in Spain: Hospital Clinic (Barcelona: coordinating centre); Hospital Sant Pau (Barcelona); Hospital Ramón y Cajal (Madrid); Hospital Gregorio Marañón (Madrid); Hospital Germans Trias y Pujol (Badalona); and Hospital Torrecardenas (Almeria).

Patients with cirrhosis admitted between February 2003 and October 2005 in any of the six participating hospitals with an endoscopy-proven oesophageal variceal bleeding were considered 
Table 1 Clinical characteristics of the patients at inclusion

\begin{tabular}{|c|c|c|}
\hline Characteristic & Drug $(n=78)$ & Drug+EBL $(n=80)$ \\
\hline Age (years) & $56(11)^{*}$ & $57(12)^{*}$ \\
\hline $\operatorname{Sex}(M / F)$ & $53 / 25$ & $65 / 15$ \\
\hline $\begin{array}{l}\text { Aetiology (alcohol/HCV/both/ } \\
\text { others) (no) }\end{array}$ & $42 / 18 / 10 / 8$ & $39 / 25 / 9 / 7$ \\
\hline Active alcoholism (\%) & 55 & 50 \\
\hline Child $\mathrm{A} / \mathrm{B} / \mathrm{C}$ patients (no) & $18 / 42 / 18$ & 16/46/18 \\
\hline Child score & $8.1(1.8)^{*}$ & $8.2(1.8)^{*}$ \\
\hline Ascites (\%) & 40 & 29 \\
\hline Total bilirubin (mg/dl) & $2.9(3.0)^{*}$ & $3.5(7.1)^{*}$ \\
\hline Serum albumin (mg/dl) & $29.1(7)^{*}$ & $28.9(5)^{*}$ \\
\hline Prothrombin ratio (\%) & $64(14)^{*}$ & $66(13)^{*}$ \\
\hline Creatinine (mg/dl) & $0.8(0.3)^{*}$ & $0.8(0.3)^{*}$ \\
\hline Heart rate $(\mathrm{bpm})$ & $85(15)^{*}$ & $85(15)^{*}$ \\
\hline Mean arterial pressure $(\mathrm{mm} \mathrm{Hg})$ & $83(11)^{*}$ & $85(12)^{*}$ \\
\hline HVPG (mm Hg) & $20.5(5)^{*}$ & $21.5(6)^{*}$ \\
\hline Large varices (\%) & 94 & 91 \\
\hline Previous variceal bleeding (\%) & 13 & 19 \\
\hline Active bleeding at endoscopy $\dagger(\%)$ & 37 & 33 \\
\hline Shock at index bled (\%) & 32 & 19 \\
\hline Haematocrit at index bled (\%) & $27(7)^{*}$ & $29(6)^{*}$ \\
\hline $\begin{array}{l}\text { Blood units transfused at index } \\
\text { bled }\end{array}$ & $2.9(2.2)^{*}$ & $2.6(2.7)^{*}$ \\
\hline \multicolumn{3}{|l|}{$\begin{array}{l}\text { Endoscopic treatment at index } \\
\text { bled }\end{array}$} \\
\hline EBL/EIS (\%) & $60 / 40$ & $57 / 43$ \\
\hline Nadolol dose (mg/day) & $102(52)^{*}$ & $90(48)^{*}$ \\
\hline IsMn dose (mg/day) & $36(9)^{*}$ & $36(10)^{*}$ \\
\hline
\end{tabular}

*Results given as the mean (SD). † $†$ Before applying endoscopic therapy. $E B L$, endoscopic band ligation; EIS, endoscopic injection sclerotherapy; HVPG, hepatic venous pressure gradient.

eligible for the study if they met the following criteria: (1) successful treatment of the index bleed with vasoactive drugs (terlipressin or somatostatin), antibiotics and endoscopic treatment; (2) age between 18 and 75 years; (3) no previous randomisation in the study; and (4) provided signed, informed, written consent to participate in the study.

Exclusion criteria were: (1) failure to fulfill entry criteria; (2) pregnancy; (3) known hepatocellular carcinoma; (4) chronic renal failure; (5) Child-Pugh score $\geqslant 13$ or a concomitant disease with reduced life expectancy; (6) contraindications to $\mathrm{BB}$ or IsMn; (7) previous treatment to prevent rebleeding with a portosystemic shunt or with combined pharmacological therapy with $\mathrm{BB}+\mathrm{IsMn}$; (8) treatment with $\mathrm{EBL}$ in the 3 months before the index bled; (9) bleeding from isolated gastric or ectopic varices; and (10) portal vein thrombosis.

\section{Randomisation}

Randomisation was performed between day 5 and day 7 of the index bleed, after at least $48 \mathrm{~h}$ of haemodynamic stability without signs of bleeding. Randomisation was stratified by Child class (A vs BC). The randomisation sequence was generated by computer in blocks of 8 and the code was kept at the coordinating centre in sealed, consecutively numbered, opaque envelopes. Randomisation was done by contacting the coordinating centre by fax.

\section{Baseline evaluation and general management}

All patients received an endoscopic treatment to control the acute bleeding episode. Before randomisation a full clinical history, physical examination, electrocardiogram, chest radiograph, laboratory tests, ultrasonography (to discard the presence of hepatocelular carcinoma or portal vein thrombosis) and hepatic vein catheterisation (with measurements of hepatic venous pressure gradient (HVPG)) were obtained according to recommended standardised procedures. ${ }^{18}$ Immediately after randomisation nadolol (Nad) was initiated. The dose was increased stepwise, every 2-3 days, up to the maximum tolerated dose or up to $240 \mathrm{mg} /$ day, and then IsMn was initiated by administering $10 \mathrm{mg}$ at night-time and increased step-wise up to a maximum of $20 \mathrm{mg}$ twice a day or the maximal tolerated dose. In general, the dose was increased if clinically tolerated, and if systolic blood pressure was $\geqslant 95 \mathrm{~mm}$ $\mathrm{Hg}$ and resting rate $>50 \mathrm{bpm}$. Patients not tolerating a dose increment were kept at the previous dose. Two weeks after being on stable doses of Nad+IsMn the HVPG was measured again.

In the group randomised to receive additional treatment with $E B L$, the first elective session was carried out within 7 days of randomisation. Then EBL sessions were scheduled every 10-14 days until variceal eradication (disappearance of varices or being too small to be sucked in the banding device). EBL sessions were performed using multiband devices; application of the bands was started at the gastro-oesophageal junction and progressed upwards in a helical way for approximately $5-8 \mathrm{~cm}$. All procedures were performed under conscious sedation with midazolam (2-4 mg, intravenously) and continuous monitoring of oxygen saturation, arterial pressure and pulse rate. Patients received proton-pump inhibitors until reaching variceal eradication. After eradication, an endoscopic control was performed at 1, 6 and 12 month intervals and then annually. If varices reappeared, further EBL sessions were initiated. Both groups of patients were visited at month 1 , month 3 and then every 3 months until the end of follow-up. Each visit included anamnesis, physical examination and blood tests including haematology and biochemistry. Ultrasonography was performed at visit 1, at month 6 and every 6 months thereafter. Patients were followed until death, liver transplantation, up to a maximum of 2 years or up to the end of the study (1 February 2006)

\section{End points}

The main endpoint of the study was recurrent bleeding from any source. Secondary endpoints were variceal rebleeding, mortality (overall and mortality related to bleeding), development of ascites, hepatorenal syndrome, hepatic encephalopathy, development of severe side-effects, blood transfusion requirements, days in hospital, use of rescue treatment to prevent rebleeding and relationship between HVPG response and rebleeding at follow-up.

All patients were instructed to come to hospital whenever they experienced melena or haematemesis. If bleeding was confirmed, an emergency endoscopy was performed. The diagnosis of variceal bleeding was done when varices were actively bleeding or had stigmata of recent bleeding and/or if fresh blood was observed in the stomach and varices were the only potential source of bleeding. Bleeding was considered to be EBL-related when endoscopy disclosed bleeding from an ulcer secondary to previous ligation. The rebleeding episode was treated primarily by means of vasoactive drugs (somatostatin or terlipressin) and endoscopic treatment, preferably with EBL. Patients with one clinically significant rebleeding (transfusion requirements of $\geqslant 2$ units of blood) or two less severe rebleedings were considered treatment failures and alternative treatments to prevent rebleeding were administered according to their physician preference. 


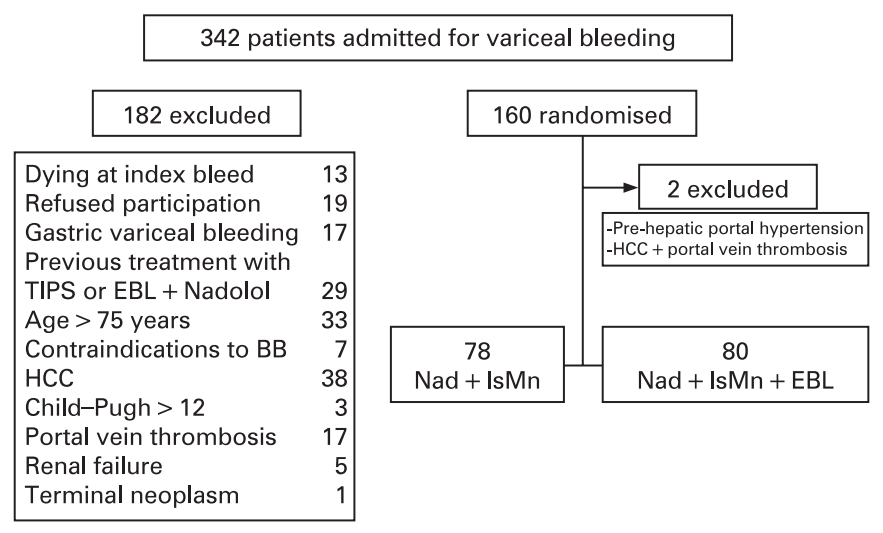

Figure 1 Enrolment of patients included in the study. BB, betablockers; EBL, endoscopic band ligation; HCC, hepatocelullar carcinoma; IsMn, isosorbide-5-mononitrate; Nad, nadolol; TIPS, transjugular intrahepatic portosystemic shunt.

\section{Sample size calculation}

The actuarial risk of recurrent bleeding at 1 year with $\mathrm{BB}+\mathrm{IsMn}$ is approximately $32 \% .^{2-5}$ The study hypothesised that by adding EBL the risk for recurrent bleeding could be decreased to $15 \%$. Because adding EBL to drug therapy would have only a rational in the case that this approach would be better than the use of drugs alone, the sample size was calculated using a one-sided test. ${ }^{19}{ }^{20}$ Seventy-six patients per group were calculated to detect the differences above mentioned with alpha $=0.05$ and beta $=0.20$.

\section{Statistical analysis}

The SPSS statistical package was used for this analysis. Data are reported as means with standard deviations. Categorical variables were compared using the Fisher's exact test. Actuarial probability curves were constructed by using the Kaplan-Meier method and compared with the log-rank test. Stepwise Cox regression analysis was used to identify independent predictors for recurrent bleeding and survival. Relative hazards and the 95\% confidence interval (CI) are given. Statistical significance was established at a $p$ value $<0.05$. The intent-to-treat strategy was used in the analysis of the results.

\section{RESULTS}

Three hundred and forty-two patients were initially considered for the study. A total of 182 patients were excluded for the reasons shown in fig 1 . Therefore, finally, 160 patients were randomised. Two patients were excluded early after randomisation, one patient because of prehepatic portal hypertension and one because of a diffuse hepatocelullar carcinoma with portal vein thrombosis; so, finally, there were 158 evaluable patients: 78 in the Nad+IsMn (Drug) group and 80 in the Nad+IsMn+EBL (Drug+EBL) group.

There were no relevant differences at entry in clinical or endoscopic characteristics (table 1).

In addition, HVPG, severity of the index bled and doses of $\mathrm{Nad}$ and IsMn administered were also similar in both groups (table 1). The use of EBL or sclerotherapy as a treatment to control acute bleeding was based in physician preferences. There were no significant differences in the severity of the bleeding episode between patients receiving EBL or sclerotherapy, as suggested by similar haematocrit at admission: 27.3 (SD 7) vs 29 (SD 6)\%; shock: $29.5 \%$ vs $24.6 \%$; blood transfusion: 2.8 (SD 2) vs 2.5 (SD 2) units (all NS). However, the finding of active

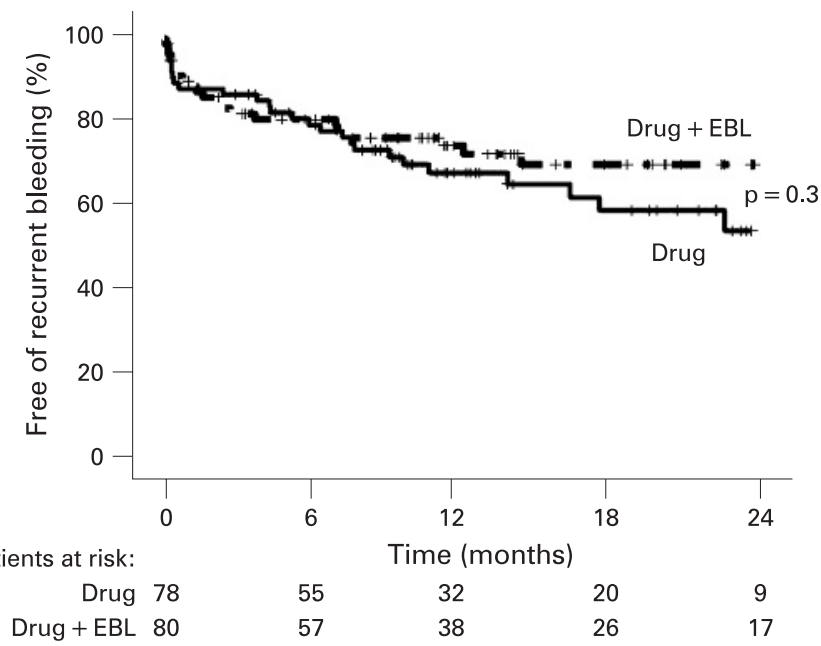

Figure 2 Actuarial probability of remaining free of recurrent bleeding from any source. EBL, endoscopic band ligation.

bleeding at the time of the procedure may have influenced the choice, since active bleeding was more frequent in patients receiving sclerotherapy than in those treated with EBL (58\% vs $28 \%)$.

Four patients, two in each group were lost to follow-up after a mean follow-up of 4 months (range: $0.3-10$ months) without developing any end-point. The remaining patients were followed until recurrent bleeding, death, liver transplantation, 2 years of follow-up or up to the end of the study. Mean followup was 14.4 (SD 7.8) months in the Drug group and 15.3 (SD 8.3) months in the Drug+EBL group (NS). Three and two patients received OLT in the Drug and in the Drug+EBL group, respectively. According to attendance to scheduled visits, scheduled EBL sessions and heart rate monitoring, $87 \%$ of patients in the Drug group and $80 \%$ of the Drug+EBL group (NS) were compliant to the allocated treatment.

Variceal eradication was achieved in 60 of the 80 patients of the Drug+EBL group after a mean of 2 (SD 1) EBL sessions and after a median of 21 days (range: 7-189). The number of additional EBL sessions to achieve eradication was not significantly different in patients receiving EBL or sclerotherapy as the acute endoscopic treatment for the index bled (1.8 (SD 1.4) vs 2.2 (SD 1.1) EBL sessions; $p=0.3$ ). In 20 of the 60 patients achieving eradication (33\%), varices reappeared after a median of 191 days (range: 54-740 days) after eradication. Variceal eradication was not achieved in 20 patients (because recurrent bleeding $(n=9)$, death $(n=3)$, lost to follow-up $(n=2)$, consent withdrawal $(n=2)$ or despite multiple EBL sessions $(n=4)$. Overall, in the Drug+EBL group 192 EBL sessions were performed and 977 bands used.

\section{Rebleeding}

Twenty-seven patients in the Drug group and 22 patients in the Drug+EBL group rebled during follow-up (NS). There were no significant differences in the 1 year $(33 \%$ vs $26 \%$; absolute risk difference (ARD): $-7 \%$; $95 \%$ CI: $-23 \%$ to $+9 \%$ ) and 2 year (46\% vs $31 \%$; ARD: $-15 \%$ : $95 \%$ CI: $-36 \%$ to $+6 \%$ ); $p=0.3$ ) actuarial probability of recurrent bleeding from any source (fig 2) In five patients recurrent bleeding was secondary to oesophageal ulcers related to endoscopic treatment (four in the Drug+EBL group and one in the Drug group) in four to portal hypertensive gastropathy (three in the Drug+EBL group and one in the Drug group) and in one, from the Drug+EBL group, no endoscopy was 
Table 2 Primary and secondary endpoints of the study according to allocated therapy

\begin{tabular}{llll}
\hline & $\begin{array}{l}\text { Drug } \\
(\mathbf{n}=\mathbf{7 8})\end{array}$ & $\begin{array}{l}\text { Drug+EBL } \\
(\mathbf{n}=\mathbf{8 0})\end{array}$ & p Value \\
\hline Rebleeding from any source & 27 & 22 & NS \\
$\quad$ Child A & 8 & 3 & 0.11 \\
$\quad$ Child B+C & 19 & 19 & NS \\
$\begin{array}{l}\text { Rebleeding from portal } \\
\text { hypertension sources }\end{array}$ & & & \\
$\quad$ Variceal rebleeding & 25 & 14 & 0.03 \\
$\quad$ Portal hypertensive gastropathy & 1 & 3 & \\
$\quad$ Post-endoscopic therapy & 1 post-EIS $\dagger$ & 4 post-EBL ulcer & \\
Source unknown & & 1 & \\
$\begin{array}{l}\text { Blood units transfused at } \\
\text { rebleeding }\end{array}$ & $4.1(4)^{*}$ & $3.6(2.4)^{*}$ & NS \\
Rescue treatments (no) & & & \\
$\quad \begin{array}{l}\text { Portosystemic shunts (TIPS/ } \\
\text { surgery) }\end{array}$ & $6 / 2$ & $9 / 0$ & \\
$\quad$ EBL \pm Nad+IsMn & 14 & 8 & \\
Days in hospital during follow-up & $10(16)^{*}$ & $11(21)^{*}$ & NS \\
Mortality & 15 & 16 & NS \\
$\quad$ Recurrent bleeding & 8 & 5 & NS \\
$\quad$ Sepsis & 3 & 2 & NS \\
Liver failure & 1 & 4 & NS \\
Others & 3 & 5 & NS \\
\hline
\end{tabular}

*Results given as the mean (SD).

$\dagger$ Endoscopic injection sclerotherapy (EIS) was used for the treatment of the index bleed.

$\mathrm{EBL}$, endoscopic variceal band ligation; ISMN, isosorbide-5-mononitrate; PHG, portal hypertensive gastropathy.

performed. (table 2)) As a consequence, when considering only patients with confirmed variceal bleeding, rebleeding was significantly higher in the Drug group than in the Drug+EBL group (table 2).

Twenty-two patients in the Drug group and 17 patients in the Drug+EBL group (NS) had treatment failure. Eight and nine patient, respectively, required derivative treatments as a rescue therapy. Other treatments received to prevent further rebleeding are depicted in table 2.

Severity of rebleeding was similar in patients treated with Drug or Drug+EBL, as shown by transfusion requirements, rescue treatments required or mortality related to bleeding (table 2). On univariate analysis, Child-Pugh class, prothrombin time, bilirubin, previous variceal bleeding, transfusion requirements and use of sclerotherapy as endoscopic treatment of the index bleed were the variables significantly related with an increased risk of recurrent bleeding. The multivariate analysis using the Cox model disclosed bilirubin (HR, 1.05; 95\% CI, 1.01 to 1.07 ), previous variceal bleeding (HR, 2.07; 95\% CI, 1.1 to 4 ), transfusion requirements (HR, 1.16; $95 \% \mathrm{CI}, 1.05$ to 1.28 ) and use of sclerotherapy (HR, 2; 95\% CI, 1.1 to 3.5 ) as independent predictors of the risk of rebleeding from any source. However, there were also no differences in recurrent bleeding between both treatment groups if patients treated with sclerotherapy were deleted from the analysis.

\section{Other complications of cirrhosis and portal hypertension}

There were no significant differences among both groups in the 1 year and 2 year actuarial probability of new or worsening ascites (Drug: 13.8\% and 20\%, Drug+EBL: 21\% and 26\%; $\mathrm{p}=0.17)$. In addition there were no significant differences in the incidence of hepatorenal syndrome ( $4.1 \%$ vs $5.3 \%$; NS), hepatic encephalopathy $(19.2 \%$ vs $22.7 \%$; NS) or hepatocellular carcinoma ( $8.6 \%$ vs $5.4 \%$; NS). There were also no significant

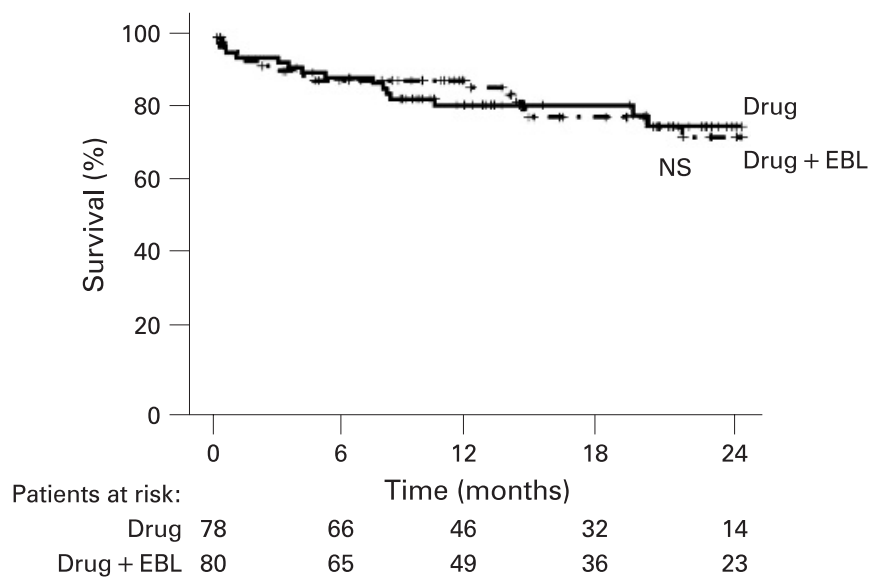

Figure 3 Actuarial probability of survival. EBL, endoscopic band ligation.

differences in days of hospitalisation for any reason during follow-up (table 2).

\section{Survival}

There were no significant differences in the 1 year and 2 year actuarial probability of survival (Drug: $82 \%$ and $77 \%$; Drug+EBL: 87 and $74 \%$, NS; fig 3). One-year ARD, $-5 \%(95 \%$ CI, $-18 \%$ to $+8 \%$ ), 2 year ARD, $+3 \%$ (95\% CI, $-18 \%$ to $+24 \%$ ). There were also no significant differences in the causes of death (table 2).

On univariate analysis, Child-Pugh class, previous encephalopathy and baseline HVPG were the variables significantly related with increased mortality. When these variables were entered on a multivariate analysis using the Cox's model, only the Child-Pugh class independently predicted mortality.

\section{Adverse effects}

Overall, the number of patients with adverse events was significantly higher in the Drug+EBL (61\%) than in the Drug group ( $32 \% ; p<0.01$ ) (table 3 ). In five patients of the Drug+EBL versus none of the Drug group the side effect was severe enough to require hospital admission $(p<0.05)($ table 3$)$. There were no significant differences in the number of patients requiring drug withdrawal (10 in the Drug+EBL vs six in the Drug group) or drug dose reduction during follow-up (12 in the Drug+EBL vs 12 in the Drug group).

\section{Clinical haemodynamic correlations}

Baseline HVPG was done in all patients. One hundred and thirtyfive patients had a second HVPG a median of 33 days after the initial HVPG. The second HVPG measurement was not done in 23 patients (13 in the Drug and 10 in the Drug+EBL group) because of early recurrent bleeding $(n=14)$, death $(n=3)$, refusal of consent $(n=2)$, early lost to follow-up $(n=1)$, failure to schedule the measurement $(n=2)$ and in one patient it was not possible to get an adequate wedged hepatic pressure due to hepatic vein-to-vein communications. Overall, 17 of the 23 patients without a second HVPG measurement rebleed during follow-up (15 from oesophageal varices) and 12 died.

In the 135 patients with repeat HVPG measurements, initial HVPG was 20.3 (SD 5) $\mathrm{mm} \mathrm{Hg}$ and decreased to 17.3 (SD 4.4) $\mathrm{mm} \mathrm{Hg}$ after beta-blockade $(12.7 \% ; \mathrm{p}<0.001)$. Forty-eight patients $(36 \%)$ had a decrease in HVPG to $<12 \mathrm{~mm} \mathrm{Hg}$ or by $>20 \%$ from baseline (responders), while these targets were not 
Table 3 Side effects observed in patients treated with Nad+IsMn or $\mathrm{Nad}+\mathrm{IsMn}+\mathrm{EBL}$

\begin{tabular}{llll}
\hline & Nad+IsMn & Nad+IsMn+EBL & p Value \\
\hline $\begin{array}{l}\text { Overall } \\
\text { Requiring withdrawal of }\end{array}$ & $25 / 78(32 \%)$ & $49 / 80(61 \%)$ & $<0.01$ \\
Nad/IsMn & & & \\
$\quad$ Hypotension & 4 & $4^{* 2}$ & \\
Impotence & 0 & 1 & \\
Bronchospasm & 1 & 0 & \\
Glaucoma & 1 & 0 & \\
Asthenia & 0 & 2 & \\
Hypoglycaemia & 0 & $2 \dagger$ & \\
Diarrhoea & 0 & $1^{* 1}$ & \\
Requiring reduction of & & & \\
Nad/IsMn dose & & & \\
Hypotension & 3 & $5^{* 5}$ & \\
Impotence & 0 & $1^{* 1}$ & \\
Asthenia & 7 & 3 & \\
Bronchospasm & 1 & 0 & \\
Bradycardia & 1 & 0 & \\
Diarrhoea & 0 & 1 & \\
Dizziness & 0 & $2^{* 2}$ & \\
No treatment modification & & & \\
Oedemas & 3 & 0 & \\
Astenia & 1 & 2 \\
Cephalea & 2 & 1 & \\
Dyspnoea & 1 & 0 & \\
Hypotension & 0 & 1 & \\
Impotence & 0 & & \\
Dizziness & 0 & & \\
& & &
\end{tabular}

Related to EBL

Post-EBL ulcer bleeding

Severe dysphagia

Glotis oedema

Asymptomatic post-EBL ulcer

* Number of patients having more than one side effect.

$\uparrow$ Requiring hospitalisation (Nad+IsMn+EBL: one hypoglycaemic coma and four postEBL ulcer bleeding).

EBL, endoscopic band ligation; IsMn, isosorbide-5-mononitrate; Nad, nadolol.

achieved in the remaining 87 patients (non-responders). For purposes of clinical hemodynamic correlations, two patients who developed complete portal vein thrombosis on follow-up were censored when complete portal vein thrombosis was detected.

One-year and 2 year actuarial probability of recurrent bleeding either from any source or from varices were significantly higher in non-responders than in responders (recurrent bleeding: $27 \%$ and $35 \%$ vs $11 \%$ and $17 \%$ : $p=0.03$; fig $4 \mathrm{~A}$; variceal rebleeding: $22 \%$ and $28 \%$ vs $5 \%$ and $10 \% ; p=0.01$ ). Importantly, there were no significant differences between nonresponders treated with Drug vs Drug+EBL with regards to 1 year and 2 year actuarial probability of rebleeding from any source (Drug: $29 \%$ and $37 \%$ vs $23 \%$ and $32 \%$ in Drug + EBL; $\mathrm{p}=0.9$; fig $4 \mathrm{~B}$ ) or from varices (Drug: $27 \%$ and $35 \%$ vs $16 \%$ and $20 \%$ in Drug $+E B L ; p=0.4)$. In addition, adding EBL did not reduce rebleeding or improve survival in HVPG-responders.

\section{DISCUSSION}

Variceal bleeding is a frequent complication of portal hypertension associated with high mortality and high risk of recurrence if no preventive treatment is initiated. ${ }^{21}$ Combined pharmacological therapy with non-selective BB plus IsMn or EBL are both considered equivalent and good alternatives in the secondary prevention of variceal bleeding. ${ }^{61}$ Based on two studies on a relatively low number of patients (122 and 80 , respectively), ${ }^{78}$ showing that patients treated with nadolol + EBL had a reduced risk of variceal rebleeding, although not of mortality, than patients treated only with EBL, it has been suggested that the combination of non-selective beta-blockers (with or without nitrates) plus EBL could be the best treatment option to prevent variceal rebleeding. ${ }^{10} 21$ This was also supported by a recent meta-analysis, ${ }^{22}$ although a recent study, only available in abstract form, ${ }^{11}$ has challenged this recommendation by showing no benefit in rebleeding rate or mortality of the combination of EBL+ propranolol over EBL alone. It should be noted, however, that these previous studies evaluated the role of adding drugs to EBL as compared with EBL alone, which does not allow the conclusion that such combination therapy is better than drug therapy alone. Because of this we aimed at investigating whether adding EBL may improve the efficacy of the combination of non-selective BB plus IsMn.

The results of the present study fail to show significant differences in the rate of recurrent bleeding among patients treated with Drug+EBL or Drugs alone. The best results were observed in Child A patients receiving Drug+EBL, the population with an expected lower rebleeding risk, although neither in this subgroup the benefit from adding EBL was statistically significant.

Recurrent bleeding was especially frequent among patients who received injection sclerotherapy as the endoscopic treatment for the index bleed. Indeed, multivariate analysis showed the use of sclerotherapy during the index bleed to be independent predictor of recurrent bleeding. It is possible that a session of sclerotherapy within the previous 10 days may favour deeper ulcers after EBL and ulcer bleeding. In addition, it has been reported that sclerotherapy produces an increase in portal pressure that is maintained during at least 5 days, while EBL only produced a transient increase in portal pressure that returns to pretreatment values within $48 \mathrm{~h} .^{23}$ This increase in portal pressure may favour portal hypertension related rebleeding. Therefore our results further support the recommendation $^{691024}$ that EBL should be the endoscopic treatment of choice during the acute bleeding episode, and suggest that avoiding sclerotherapy during the index bleed may reduce the incidence of non-variceal rebleeding episodes.

When only patients with confirmed variceal rebleeding were considered (a secondary end-point of this study), patients treated with Drug+EBL had a lower probability of rebleeding than patients treated with Drug alone. It is important to remark, however, that in clinical practice, it is often difficult to distinguish between bleeding from varices or bleeding from ligation ulcers. Even if, theoretically, it should be possible to correctly distinguish these two events, the clinical relevance of this is limited: both are due to portal hypertension and may be life-threatening for the patient. A similar situation applies to portal hypertensive gastropathy. Moreover, the possible slight benefit in preventing variceal rebleeding of adding EBL to drug therapy was at the expense of having more side effects, some of them requiring hospital admission (mainly due to rebleeding from oesophageal ulcers), and of a huge number of endoscopic procedures to perform the scheduled EBL sessions and to evaluate variceal recurrence. This makes very likely a greater cost in the group Drug+EBL than in the group treated with Drug only.

Important enough, there were no significant differences in survival among patients treated with Drugs+EBL or Drugs 
A

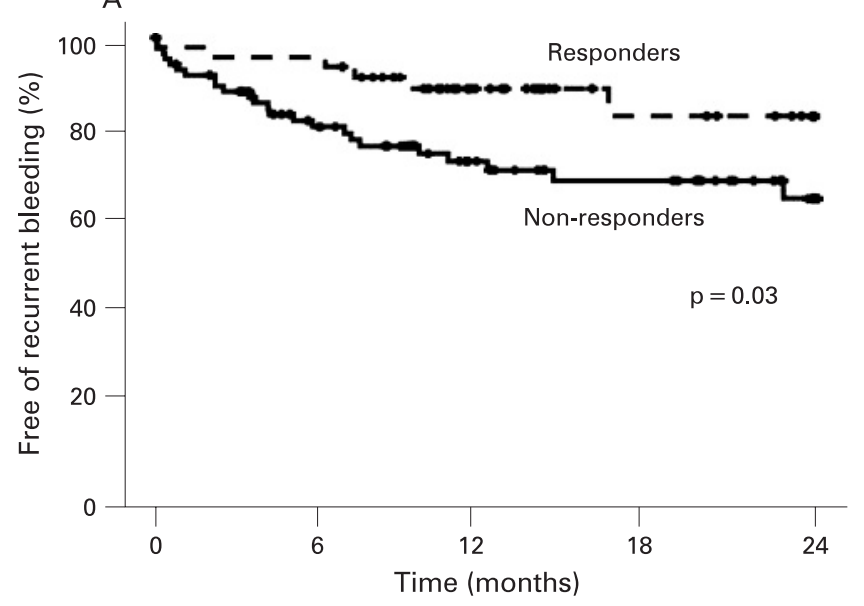

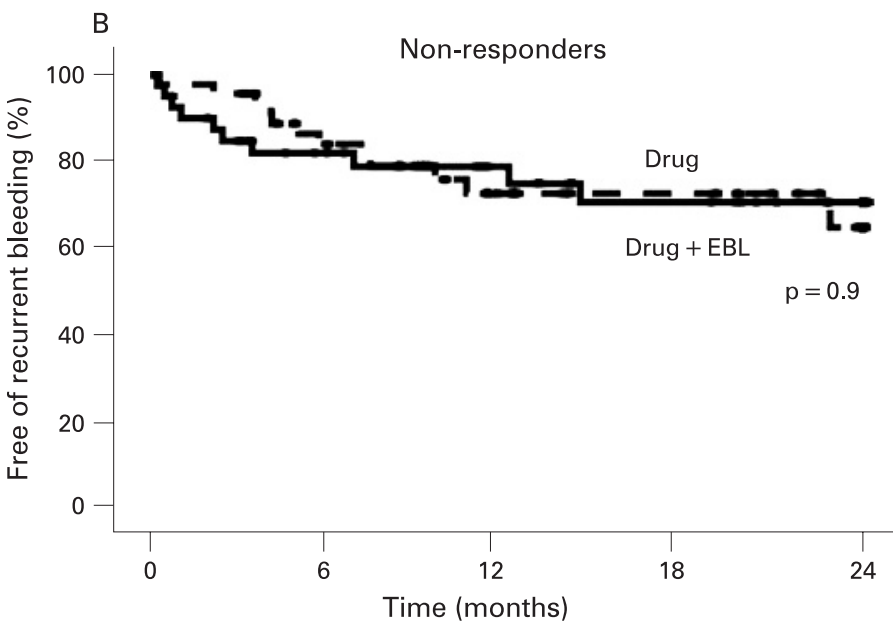

Figure 4 (A) Actuarial probability of recurrent bleeding from any source in patients showing a reduction in the HVPG below $12 \mathrm{~mm} H g$ or more than $20 \%$ (responders) and in those not achieving these targets (non-responders). (B) Actuarial probability of recurrent bleeding from any source in patients not achieving a reduction in the HVPG below $12 \mathrm{~mm} \mathrm{Hg}$ or more than 20\% (HVPG non-responders) treated with nadolol + isosorbide-5-mononitrate (Drug) or Drug + endoscopic band ligation (EBL). HVPG, hepatic venous pressure gradient.

alone. Indeed, the degree of liver failure, evaluated by the Child-Pugh classification, was the only independent predictor of survival.

The results of the present study confirm that HVPG responders exhibit a significantly lower rebleeding rate. ${ }^{112}$ However, and contrary to what has been suggested, adding EBL to drug therapy failed to reduce the risk of recurrent bleeding. These findings are in agreement with previous observations already suggesting that adding EBL may not be the optimal approach to improve the outcome of HVPG nonresponders ${ }^{217} 25$ and support the view that HVPG non-responders are a special high-risk population that may benefit from a more aggressive approach, such as an early decision for TIPS. This, however, should be evaluated in RCTs.

In summary, the results of the present study show that adding EBL to pharmacological treatment fails to significantly reduce the incidence of recurrent bleeding, the need of rescue therapy or mortality, and is associated with more adverse events and probably higher costs that the use of pharmacological treatment alone. Associating EBL to drug therapy did not modify survival and did not improve the worse prognosis of HVPG non-responders.

\section{Spanish Variceal Bleeding Study Group}

Members of the Spanish Variceal Bleeding Study Group contributing to the study were:

Steering committee: JC Garcia-Pagán, C Villanueva, J Bosch

Data Monitoring Committee: JG Abraldes, R Saez

Clinical Evaluation Committee: JC Garcia-Pagán, C Villanueva, A Albillos, R Bañares, R Planas, J Bosch

Patient recruitment: Hospital Clinic, Barcelona: A Escorsell, P Bellot, J Turnes, J Llach. Hospital de Sant Pau, Barcelona: C Aracil, A Colomo, X Torres. Hospital Universitario Ramón y Cajal, Madrid: M González, R González, C Martín. Hospital Gregorio Marañón, Madrid: M-V Catalina, C Ripoll. Hospital Germans Trias i Pujol, Badalona: J Boix, R Planas. Department of Gastroenterology, Hospital Torrecardenas, Almeria: M Casado.

Funding: Supported in part by grants from Instituto de Salud Carlos III (FIS 04/0655 and 06/0623). Ciberehd is funded by Instituto de Salud Carlos III. Nadolol was kindly supplied by Sanofi Winthrop (Barcelona, Spain). Isosorbide mononitrate was kindly provided by Lacer (Barcelona, Spain).
Competing interests: None.

Ethics approval: The study protocol was approved by the Ethics Committees of all participating hospitals and by the Ministry of Health, and fulfilled the guidelines of Good Clinical Practice in clinical trials.

\section{REFERENCES}

1. Bosch J, Garcia-Pagan J. Prevention of variceal rebleeding. Lancet 2003;361:952-4.

2. Villanueva C, Minana J, Ortiz J, et al. Endoscopic ligation compared with combined treatment with nadolol and isosorbide mononitrate to prevent recurrent variceal bleeding. N Engl J Med 2001;345:647-55.

3. Patch D, Sabin CA, Goulis J, et al. A randomized, controlled trial of medical therapy versus endoscopic ligation for the prevention of variceal rebleeding in patients with cirrhosis. Gastroenterology 2002;123:1013-9.

4. Lo GH, Chen WC, Chen MH, et al. Banding ligation versus nadolol and isosorbide mononitrate for the prevention of esophageal variceal rebleeding. Gastroenterology 2002;123:728-34.

5. Romero G, Kravetz D, Argonz J, et al. Comparative study between nadolol and 5isosorbide mononitrate vs. endoscopic band ligation plus sclerotherapy in the prevention of variceal rebleeding in cirrhotic patients: a randomized controlled trial. Aliment Pharmacol Ther 2006:24:601-11.

6. de Franchis R. Evolving Consensus in Portal Hypertension: Report of the Baveno IV Consensus Workshop on methodology of diagnosis and therapy in portal hypertension. J Hepatol 2005;43:167-76.

7. Lo GH, Lai KH, Cheng JS, et al. Endoscopic variceal ligation plus nadolol and sucralfate compared with ligation alone for the prevention of variceal rebleeding: a prospective, randomized trial. Hepatology 2000;32:461-5.

8. de la PJ, Brullet E, Sanchez-Hernandez E, et al. Variceal ligation plus nadolol compared with ligation for prophylaxis of variceal rebleeding: a multicenter trial. Hepatology 2005;41:572-8.

9. Garcia-Tsao G, Sanyal AJ, Grace ND, et al. Prevention and management of gastroesophageal varices and variceal hemorrhage in cirrhosis. Am J Gastroenterol 2007; 102:2086-102.

10. Garcia-Tsao G, Sanyal AJ, Grace ND, et al. Prevention and management of gastroesophageal varices and variceal hemorrhage in cirrhosis. Hepatology 2007:46:922-38.

11. Jha SK, Kumar A, Sharma BC, et al. Endoscopic variceal ligation (EVL) plus propranolol (P) and isosorbide mononitrate (ISMN) versus EVL alone in secondary prophylaxis of variceal bleeding: a prospective RCT. Hepatology 2007;46(suppl 1):250A.

12. D'Amico G, Garcia-Pagan JC, Luca A, et al. Hepatic vein pressure gradient reduction and prevention of variceal bleeding in cirrhosis: a systematic review. Gastroenterology 2006;131:1611-24.

13. Feu F, Garcia-Pagan JC, Bosch J, et al. Relation between portal pressure response to pharmacotherapy and risk of recurrent variceal haemorrhage in patients with cirrhosis. Lancet 1995;346:1056-9.

14. Abraldes JG, Tarantino I, Turnes J, et al. Hemodynamic response to pharmacological treatment of portal hypertension and long-term prognosis of cirrhosis. Hepatology 2003;37:902-8.

15. Garcia-Pagan JC, Navasa M, Bosch J, et al. Enhancement of portal pressure reduction by the association of isosorbide-5-mononitrate to propranolol administration in patients with cirrhosis. Hepatology 1990;11:230-8. 
16. Morillas RM, Planas R, Cabre E, et al. Propranolol plus isosorbide-5-mononitrate for portal hypertension in cirrhosis: long-term hemodynamic and renal effects. Hepatology 1994;20:1502-8.

17. Villanueva C, Balanzo J, Novella MT, et al. Nadolol plus isosorbide mononitrate compared with sclerotherapy for the prevention of variceal rebleeding. N Engl J Med 1996;334:1624-9.

18. Bosch J, Garcia-Pagan JC, Berzigotti A, et al. Measurement of portal pressure and its role in the management of chronic liver disease. Semin Liver Dis 2006;26:348-62.

19. Dunnett CW, Gent M. An alternative to the use of two-sided tests in clinical trials. Stat Med 1996;15:1729-38.

20. Sackett DL. The principles behind the tactics of performing therapeutic trials. In: Haynes B, Sackett DL, Guyatt G, Tulliez M, eds. Clinical epidemiology. Lippincott Williams \& Wikins, 2006: 173-243.
21. Bosch J, Abraldes JG, Berzigotti A, et al. Portal hypertension and gastrointestinal bleeding. Semin Liver Dis 2008;28:3-25.

22. Gonzalez R, Zamora J, Gomez-Camarero J, et al. Meta-analysis: Combination endoscopic and drug therapy to prevent variceal rebleeding in cirrhosis. Ann Intern Med 2008;149:109-22.

23. Avgerinos A, Armonis A, Stefanidis G, et al. Sustained rise of portal pressure after sclerotherapy, but not band ligation, in acute variceal bleeding in cirrhosis. Hepatology 2004;39:1623-30.

24. Villanueva C, Piqueras $\mathrm{M}$, Aracil C, et al. A randomized controlled trial comparing ligation and sclerotherapy as emergency endoscopic treatment added to somatostatin in acute variceal bleeding. J Hepatol 2006;45:560-7.

25. Bureau C, Peron JM, Alric L, et al. "A la carte" treatment of portal hypertension: adapting medical therapy to hemodynamic response for the prevention of bleeding. Hepatology 2002;36:1361-6.

\section{BMJ Careers Fair}

\section{2-3 October 2009, Business Design Centre, London, UK}

\section{9-10 October 2009, Thinktank, Birmingham, UK}

$\mathrm{BMJ}$ is the largest organiser of medical recruitment fairs across the UK. This year we are organising two careers fairs, in partnership with the London Deanery on 2-3 October in London, and the West Midlands Deanery on 9-10 October in Birmingham.

Whatever your grade or specialty there is a careers fair for you. You can:

- attend seminars on topics such as CV writing, interview skills, planning your career and working abroad

- visit exhibition stands to get careers advice, find a new job, identify alternative career pathways

It's free to attend the exhibition if you register online in advance. There is a small fee for attending our seminar programme.

Register online today at www.careersfair.bmj.com 
Nadolol plus isosorbide mononitrate alone or associated with band ligation in the prevention of recurrent bleeding: a multicentre randomised controlled trial

J C García-Pagán, C Villanueva, A Albillos, et al.

Gut 2009 58: 1144-1150 originally published online February 12, 2009 doi: 10.1136/gut.2008.171207

Updated information and services can be found at:

http://gut.bmj.com/content/58/8/1144.full.html

\section{These include:}

References This article cites 24 articles, 1 of which can be accessed free at: http://gut.bmj.com/content/58/8/1144.full.html\#ref-list-1

Article cited in:

http://gut.bmj.com/content/58/8/1144.full.html\#related-urls

Email alerting

Receive free email alerts when new articles cite this article. Sign up in service the box at the top right corner of the online article.

Notes

To request permissions go to:

http://group.bmj.com/group/rights-licensing/permissions

To order reprints go to:

http://journals.bmj.com/cgi/reprintform

To subscribe to BMJ go to:

http://group.bmj.com/subscribe/ 